This document is confidential and is proprietary to the American Chemical Society and its authors. Do not copy or disclose without written permission. If you have received this item in error, notify the sender and delete all copies.

\title{
One-pot synthesis of enantiopure pyrrolopiperazines
}

\begin{tabular}{|r|l|}
\hline Journal: & The Journal of Organic Chemistry \\
\hline Manuscript ID & jo-2020-02103v.R1 \\
\hline Manuscript Type: & Note \\
\hline Date Submitted by the \\
Author: & n/a \\
\hline Complete List of Authors: & $\begin{array}{l}\text { Pertejo, Pablo; Universidad de Burgos Facultad de Ciencias } \\
\text { González-Saiz, Beatriz; Universidad de Burgos Facultad de Ciencias } \\
\text { Quesada, Roberto; Universidad de Burgos, Química } \\
\text { Garcia-Valverde, Maria; Universidad de Burgos, Department of } \\
\text { Chemistry }\end{array}$ \\
\hline
\end{tabular}




\section{Introduction}

Piperazines and pyrrolopiperazines represent privileged scaffolds in medicinal chemistry. Their drug-like properties and synthetic versatility make them excellent candidates to develop ligands for diverse protein receptors in drug discovery programs. ${ }^{1}$ Thus, these heterocyclic structures are found in many drugs and drug candidates with different pharmacological activity (Figure 1). ${ }^{2} \mathrm{~A}$ large number of methodologies have been described for the synthesis of these heterocycles, many of them starting from non-protected 1,2-diamines, such as the reductive amination of dicarbonyl compounds, ${ }^{3}$ the cyclocondensation with diols via borrowing hydrogen, ${ }^{4}$ the reductive coupling of diimines, ${ }^{5}$ the diamination of allenes ${ }^{6}$ or the Michael addition-based multicomponent domino reaction involving 1,3-dicarbonyl compounds. ${ }^{7}$

Figure 1. Drugs containing piperazine scaffold
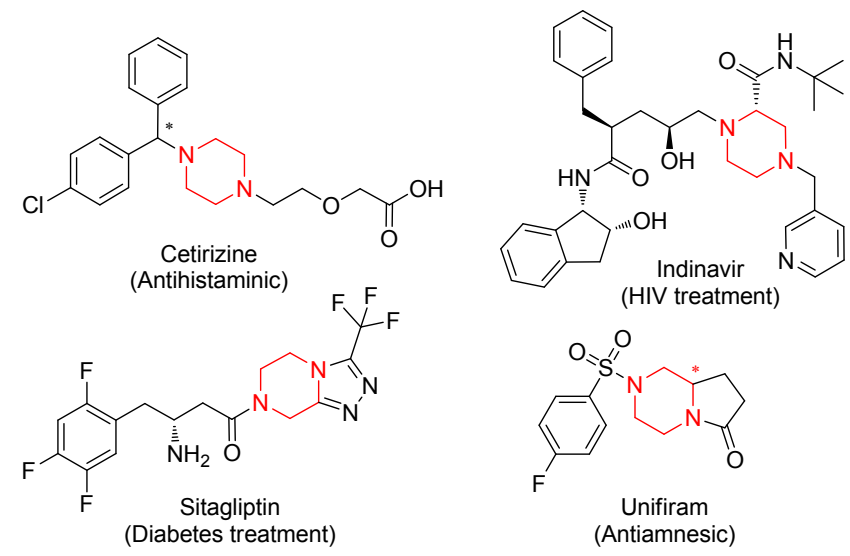

Within the realm of multicomponent reactions (MCR), ${ }^{8}$ those based on the isocyanide chemistry (IMCR) ${ }^{9}$ have demonstrated the greatest versatility in the synthesis of piperazines, ${ }^{10}$

although the use of non-protected diamines in IMCR is rather limited. In fact, the use of non-protected primary diamines in the classical Ugi four component reaction (U-4CR) has been mainly focused in the construction of polymers ${ }^{11}$ and macrocycles. ${ }^{12}$ Actually, the synthesis of small molecules using diamines in the Ugi-4CR usually requires protecting groups in order to avoid competitive reactions. ${ }^{13}$ Thus, the use of primary non-protected 1,2-diamines usually affords 2-aminopyrazines as a result of an interrupted Ugi reaction, ${ }^{14}$ where the nitrilium ion, intermediate in the Ugi reaction, reacts intramolecularly with the second amine group of the diamine avoiding the incorporation of the carboxylic component. Furthermore, when secondary non-protected diamines are employed in the Ugi reaction, the four components are integrated in the final product with a different connectivity to the classical Ugi 4-CR adduct, the split-Ugi adduct. ${ }^{15}$ In this case, although the carboxylic component is incorporated and the imino anhydride intermediate is generated, the expected Mumm rearrangement does not take place since the nitrogen atom involved in the Ugi reaction becomes a tertiary amine. In this situation the second secondary amine provokes the rearrangement leading to the migration of the acyl moiety to this position. For all these reasons, there are only a handful of examples of classical Ugi reaction with non-protected diamines, ${ }^{16}$ all of them sharing a common feature, the use of functionalized aldehydes in order to block the second amine group in the first stage of the reaction. In an effort to increase the applicability of non-protected diamines in the synthesis of piperazines through the Ugi-4CR, we envisaged the combination of non-protected 1,2-diamines with arylglyoxals (Scheme 1).

Scheme 1. Primary non-protected diamines in the Ugi reaction 
1. Interrupted Ugi reaction

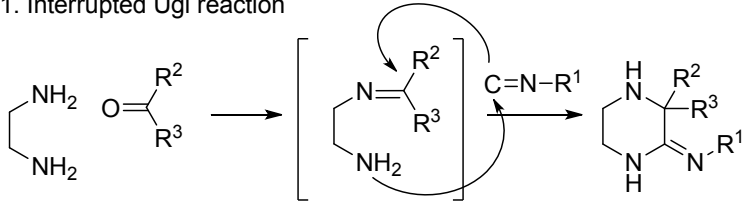

2. Functionalized aldehydes and diamines in the Ugi reaction

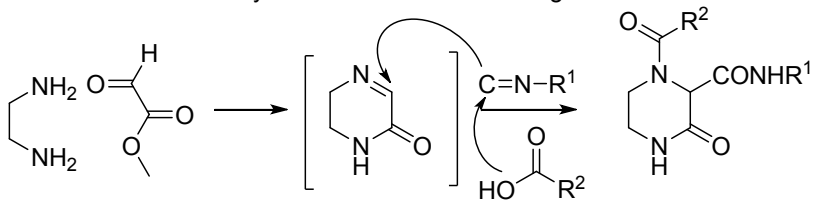

3. This work: One step synthesis of pyrrolopiperazines

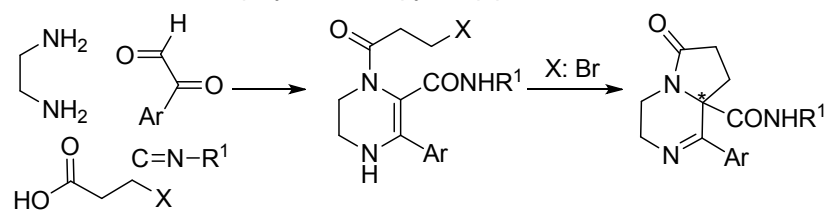

\section{Results and discussion}

Initially, we decided to use ethylendiamine 1a, phenylglyoxal 2a, acetic acid 3a and cyclohexyl isocyanide 4a as starting reactants. The Ugi reaction was carried out under standard conditions,${ }^{17}$ therefore the diamine $1 \mathbf{a}$ (1 equiv) was mixed with a solution of phenylglyoxal 2a (1 equiv) in methanol. The cyclohexyl isocyanide 4a (1 equiv) and the acetic acid 3a (1 equiv) were then added and the mixture was stirred at room temperature for one day. After work up, the reaction mixture was analysed by NMR techniques, being the piperazine $\mathbf{5}$ the only product observed, as a mixture of imine/enamine tautomers (Scheme 2, see Supporting Information). Furthermore, the result was identical to the one obtained when we synthesized and isolated the diimine ( $\mathbf{A}$ in scheme 3 ) from the ethylenediamine 1a and the phenylglyoxal $\mathbf{2 a},{ }^{18}$ and then it was treated with the isocyanide $\mathbf{4 a}$ and the carboxylic acid $\mathbf{3 a}$, showing that this diimine $\mathbf{A}$ was an intermediate in this synthesis.

Scheme 2. One-pot synthesis of piperazines from nonprotected ethylenediamine<smiles>NCCN</smiles>

1a<smiles>CC(=O)O</smiles>

3a<smiles>O=CC(=O)c1ccccc1</smiles>

2a<smiles></smiles>

$4 a$

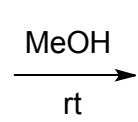

rt

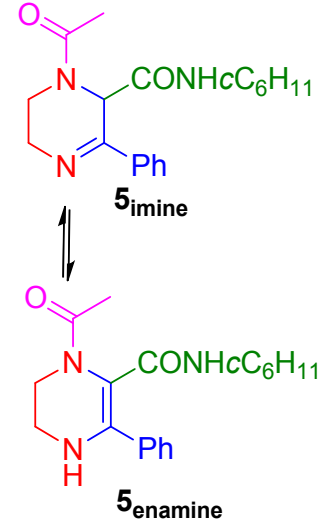

This result prompted us to explore the characteristic reactivity of enamines as nucleophiles, ${ }^{19}$ introducing an electrophilic position on the carboxylic acid component in order to furnish a fused lactam with the piperazine system. We chose 3bromopropionic acid $\mathbf{3 b}$ and the reaction was carried out in the conditions described above. Gratefully, the cyclization to $\gamma$ lactam took place spontaneously, ${ }^{20}$ yielding the corresponding pyrrolopiperazine 6a (Table 1, Entry 1) through a multicomponent-domino reaction. In order to determine the scope of this reaction, we also used the 2,3-dimethyl-2,3butanediamine $\mathbf{1 b}$, along with different arylglyoxals $\mathbf{2 a - b}$ and isocyanides $\mathbf{4 a - c}$ affording different pyrrolopiperazines 6a-f. We observed lower yields when the hindered diamine $\mathbf{1 b}$ was used (Entries 4-6 vs 1-3, Table 1).

Table 1. One-pot synthesis of pyrrolopiperazines 6 from non-protected 1,2-diamines

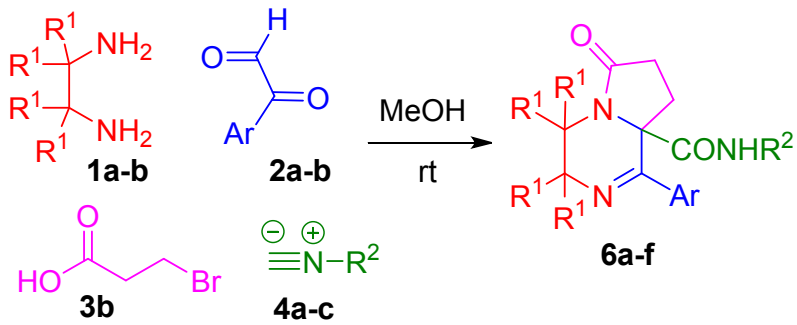

$\begin{array}{ccccc}\text { Entry } & \mathbf{1}\left(\mathbf{R}^{\mathbf{1}}\right) & \mathbf{2}(\mathbf{A r}) & \mathbf{4}\left(\mathbf{R}^{\mathbf{2}}\right) & \mathbf{6}(\mathbf{\%}) \\ 1 & \mathbf{1 a}(\mathrm{H}) & \mathbf{2 a}\left(\mathrm{C}_{6} \mathrm{H}_{5}\right) & \mathbf{4 a}\left(c \mathrm{C}_{6} \mathrm{H}_{11}\right) & \mathbf{6 a}(92) \\ 2 & \mathbf{1 a}(\mathrm{H}) & \mathbf{2 a}\left(\mathrm{C}_{6} \mathrm{H}_{5}\right) & \mathbf{4 b}(t \mathrm{Bu}) & \mathbf{6 b}(88) \\ 3 & \mathbf{1 a}(\mathrm{H}) & \mathbf{2 b}\left(4-\mathrm{ClC}_{6} \mathrm{H}_{4}\right) & \mathbf{4 a}\left(c \mathrm{C}_{6} \mathrm{H}_{11}\right) & \mathbf{6 c}(89) \\ 4 & \mathbf{1 b}\left(\mathrm{CH}_{3}\right) & \mathbf{2 a}\left(\mathrm{C}_{6} \mathrm{H}_{5}\right) & \mathbf{4 a}\left(c \mathrm{C}_{6} \mathrm{H}_{11}\right) & \mathbf{6 d}(72) \\ 5 & \mathbf{1 b}\left(\mathrm{CH}_{3}\right) & \mathbf{2 a}\left(\mathrm{C}_{6} \mathrm{H}_{5}\right) & \mathbf{4 b}(t \mathrm{Bu}) & \mathbf{6 e}(58) \\ 6 & \mathbf{1 b}\left(\mathrm{CH}_{3}\right) & \mathbf{2 a}\left(\mathrm{C}_{6} \mathrm{H}_{5}\right) & \mathbf{4 c}(\mathrm{nBu}) & \mathbf{6 f}(68)\end{array}$

a. Isolated products yields

To account for this result, we proposed a mechanistic pathway starting from the cyclic diimine $\mathbf{A}$. The complete chemoselectivity observed in the following step can be explained by the different reactivity of these imines. Thus, the Ugi reaction would take place exclusively on the aldimine, while the aromatic ketimine would remain unchanged, affording selectively the imino anhydride intermediate $\mathbf{B}$. The Mumm rearrangement would yield the corresponding Ugi adduct, a cyclic imine $\mathbf{C}_{\text {imine }}$ in equilibrium with its tautomer, the cyclic enamine $\mathbf{C}_{\text {enamine. }}$. In the last step the enamine would react spontaneously with formal $\mathrm{HBr}$ elimination yielding the corresponding pyrrolopiperazine 6 (Scheme 3).

Scheme 3. Proposed mechanistic pathway

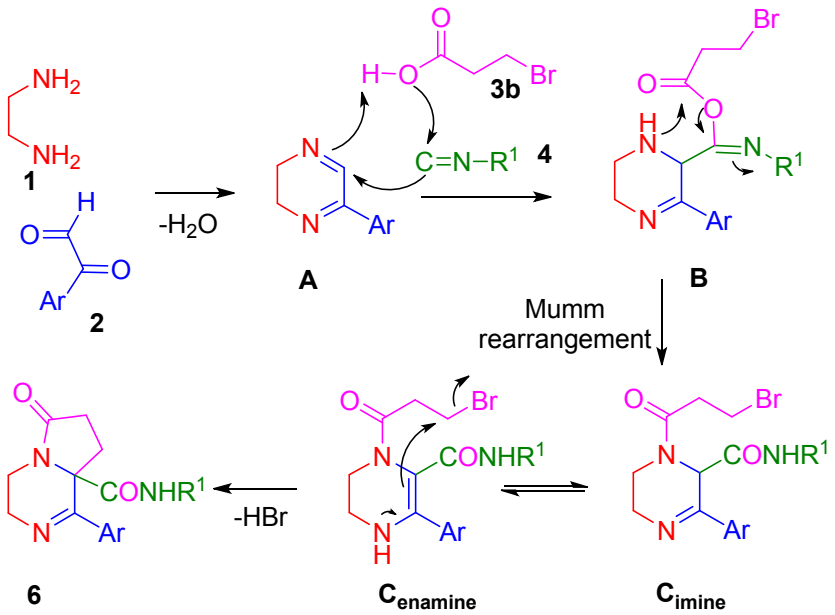

Interestingly, the new stereogenic center is created in the last cyclization step and not during the Ugi reaction. ${ }^{21}$ This is remarkable, since the stereoselectivity achieved in the Ugi reaction is usually poor. ${ }^{22}$ Thus, we attempted the 
stereochemical control in the generation of the new stereogenic center by using symmetric diamine derivatives bearing chiral centres. Enantiopure diamines, (1S,2S)-1,2cyclohexanediamine $\mathbf{1 c}$ and (1S,2S)-1,2diphenylethylenediamine 1d, and meso-diamines, cisdiaminocyclohexane $\mathbf{1 e}$ and meso-1,2diphenylethylenediamine 1f were assayed employing the same protocol described above, and the corresponding pyrrolopiperazines $\mathbf{7 a - j}$ were obtained with total diastereoselectivity in excellent yields, regardless of the nature of the diamine employed (Scheme 4). The configuration of the new stereogenic centers was determined by NOESY experiments. Moreover, structural analyses by X-ray diffraction of compounds $7 \mathrm{a}^{23}$ and $\mathbf{7 g}$ confirmed the determined stereochemistry (see Supporting Information).

Scheme 4. One-pot diastereoselective synthesis of pyrrolopyrazines 7
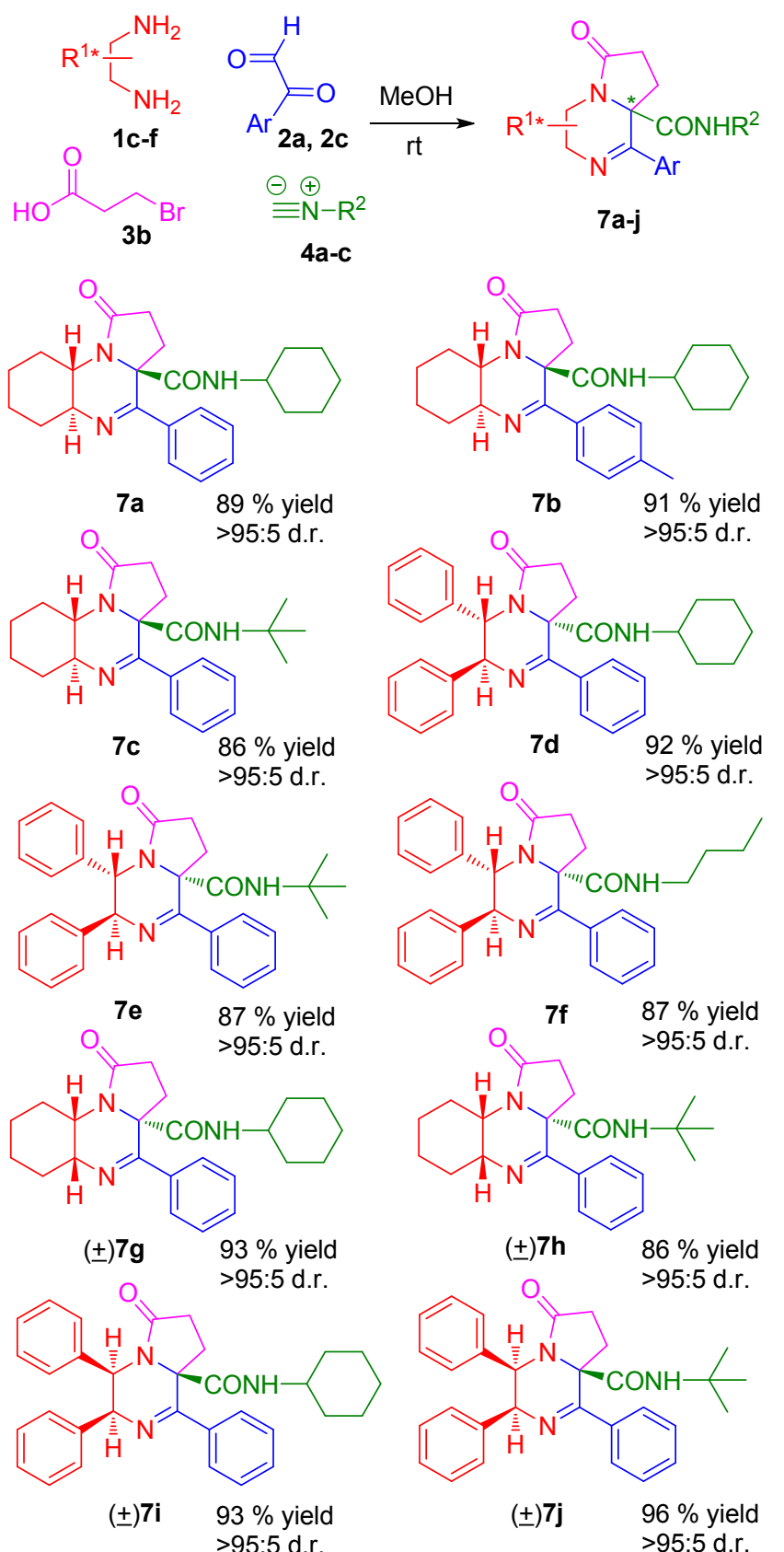

The complete diastereoselectivity observed in the last cyclization step was also studied computationally. This result can be explained by the reaction through the preferred conformations in the cyclic enamine intermediate, determined by DFT calculations using Gaussian 09. ${ }^{24}$ Thus, in the synthesis of 7a, the most stable conformation of the enamine intermediate favors the $S i$ face attack of the enamine, while for the synthesis of $7 \mathbf{d}, 7 \mathrm{~g}$ and $7 \mathbf{i}$ the preferred conformation favors the $R e$ face attack (Figure 2, see Supporting Information).

Figure 2. Energies calculated (in gas phase) for the most stable conformations of the enamine intermediate in the synthesis of $7 \mathrm{a}$ and $7 \mathrm{~g}$
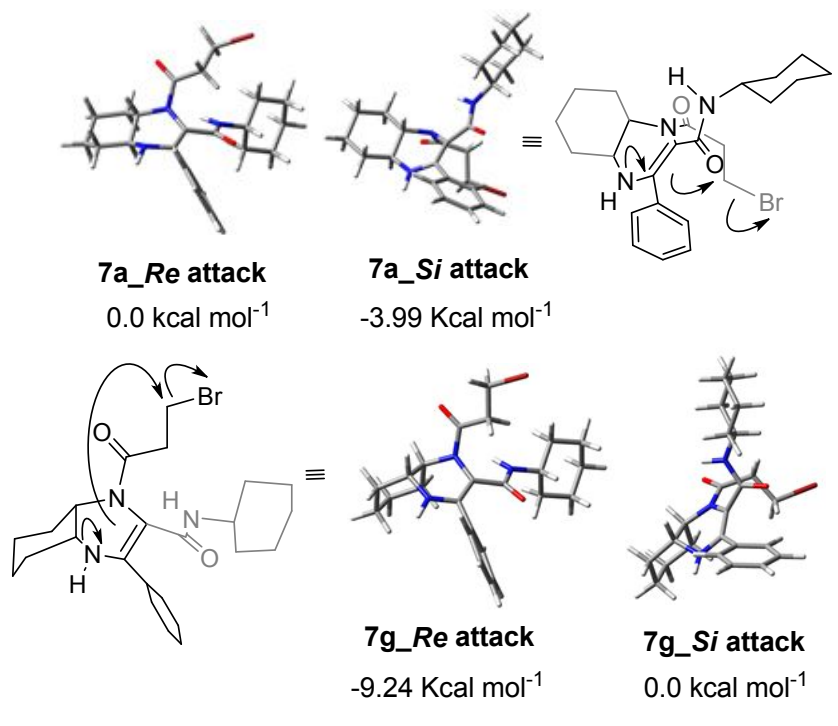

\section{Conclusion}

In this work we have developed an efficient and simple methodology for the synthesis of enantiopure pyrrolopiperazines through a multicomponent domino reaction, Ugi/enamine alkylation, using non-protected diamines in a onepot sequence with high yield, and total diastereoselectivity when diamines bearing stereogenic centers are used.

\section{Experimental section}

General methods. Melting points are not corrected. Optical rotations were measured on a Zeiss D-7082 Polarimeter in a 1 $\mathrm{dm}$ cell and concentrations are given in $\mathrm{g} / 100 \mathrm{ml} .{ }^{1} \mathrm{H}$ and ${ }^{13} \mathrm{C}$ NMR spectra were recorded in $\mathrm{CDCl}_{3}$ at 300 and $75 \mathrm{MHz}$, respectively, on a Varian Mercury 300 system, DEPT-135 experiments were conducted to assign carbon-13 signals. Chemical shifts are reported in parts per million with respect to residual solvent protons, and coupling constants are reported in hertz. High resolution mass spectra were recorded in on a 6545 Q-TOF Agilent LC-MS mass spectrometer (positive electrospray ionization mode, ESI $(+))$.

General procedure for the synthesis of pyrrolopiperazines 6 and 7. The corresponding diamine 1a-d $(1 \mathrm{mmol})$ was added to a solution of arylglyoxal 2a-c $(1 \mathrm{mmol})$ in methanol $(10 \mathrm{ml})$. The solution was stirred for $10 \mathrm{~min}$ at room temperature. Then the 3-bromopropionic acid $\mathbf{3 b}(1 \mathrm{mmol})$ and the isocyanide $\mathbf{2 a - b}$ $(1 \mathrm{mmol})$ were added to the solution and the mixture was stirred for $24 \mathrm{~h}$. Then the solvent was removed and the residue was dissolved in dichloromethane and washed first with $10 \%$ 
aqueous $\mathrm{HCl}$ solution, and then with saturated aqueous $\mathrm{Na}_{2} \mathrm{CO}_{3}$ solution. The organic phase was dried over anhydrous $\mathrm{Na}_{2} \mathrm{SO}_{4}$, filtered and concentrated to dryness. The residue was purified by column chromatography.

N-Cyclohexyl-6-oxo-1-phenyl-3,4,6,7,8,8a-

hexahydropyrrolo[1,2-a]pyrazine-8a-carboxamide (6a). White solid (Hexane/AcOEt, 2:1). Yield: $312 \mathrm{mg}, 92 \%$. M. p. 158$160{ }^{\circ} \mathrm{C} .{ }^{1} \mathrm{H} \mathrm{NMR}\left(300 \mathrm{MHz}, \mathrm{CDCl}_{3}\right) \delta: 7.70-7.66(\mathrm{~m}, 2 \mathrm{H}), 7.37-$ $7.30(\mathrm{~m}, 3 \mathrm{H}), 6.00(\mathrm{~d}, J=8.3 \mathrm{~Hz}, 1 \mathrm{H}), 3.99-3.72(\mathrm{~m}, 4 \mathrm{H}), 3.23$ $3.13(\mathrm{~m}, 1 \mathrm{H}), 2.86-2.67(\mathrm{~m}, 2 \mathrm{H}), 2.27-2.19(\mathrm{~m}, 1 \mathrm{H}), 1.94-0.93$ $(\mathrm{m}, 11 \mathrm{H}) \cdot{ }^{13} \mathrm{C}\left\{{ }^{1} \mathrm{H}\right\} \mathrm{NMR}\left(75 \mathrm{MHz}, \mathrm{CDCl}_{3}\right) \delta: 173.3(\mathrm{Cq}), 168.1$ $(\mathrm{Cq}), 166.5(\mathrm{Cq}), 135.8(\mathrm{Cq}), 130.4\left(\mathrm{CH}_{\mathrm{Ar}}\right), 128.4\left(\mathrm{CH}_{\mathrm{Ar}}\right), 127.6$ $\left(\mathrm{CH}_{\mathrm{Ar}}\right), 66.6(\mathrm{Cq}), 49.4(\mathrm{CH}), 47.5\left(\mathrm{CH}_{2}\right), 34.4\left(\mathrm{CH}_{2}\right), 32.8$ $\left(\mathrm{CH}_{2}\right), 32.4\left(\mathrm{CH}_{2}\right), 30.7\left(\mathrm{CH}_{2}\right), 29.9\left(\mathrm{CH}_{2}\right), 25.2\left(\mathrm{CH}_{2}\right), 24.9$ $\left(\mathrm{CH}_{2}\right), 24.7\left(\mathrm{CH}_{2}\right)$. HRMS (ESI) $\mathrm{m} / \mathrm{z}:[\mathrm{M}+\mathrm{H}]^{+}$Calcd for $\mathrm{C}_{20} \mathrm{H}_{26} \mathrm{~N}_{3} \mathrm{O}_{2} 340.2020$; Found 340.2022

$N$-(tert-Butyl)-6-oxo-1-phenyl-3,4,6,7,8,8a-

hexahydropyrrolo [1,2-a]pyrazine-8a-carboxamide.

(6b). Brown solid (Hexane/AcOEt, 2:1). Yield: 275 mg, 88 \%. M. p. 106-108 ${ }^{\circ} \mathrm{C} .{ }^{1} \mathrm{H}$ NMR $\left(300 \mathrm{MHz}, \mathrm{CDCl}_{3}\right) \delta$ 7.75-7.63 (m, $\left.2 \mathrm{H}\right)$, 7.43-7.31 (m, 3H), $5.52(\mathrm{~s}, 1 \mathrm{H}), 4.10-3.94(\mathrm{~m}, 2 \mathrm{H}), 3.91-3.80$ $(\mathrm{m}, 1 \mathrm{H}), 3.26-3.12(\mathrm{~m}, 1 \mathrm{H}), 2.91-2.70(\mathrm{~m}, 2 \mathrm{H}), 2.35-2.11(\mathrm{~m}$, $1 \mathrm{H}), 1.94-1.82(\mathrm{~m}, 1 \mathrm{H}), 1.28(\mathrm{~s}, 9 \mathrm{H}) .{ }^{13} \mathrm{C}\left\{{ }^{1} \mathrm{H}\right\}$ NMR $(75 \mathrm{MHz}$, $\left.\mathrm{CDCl}_{3}\right) \delta 173.3(\mathrm{Cq}), 168.1(\mathrm{Cq}), 166.2(\mathrm{Cq}), 135.6(\mathrm{Cq}), 130.5$ $\left(\mathrm{CH}_{\mathrm{Ar}}\right), 128.5\left(\mathrm{CH}_{\mathrm{Ar}}\right), 127.5\left(\mathrm{CH}_{\mathrm{Ar}}\right), 67.2(\mathrm{Cq}), 52.5(\mathrm{Cq}), 47.7$ $\left(\mathrm{CH}_{2}\right), 34.3\left(\mathrm{CH}_{2}\right), 30.8\left(\mathrm{CH}_{2}\right), 29.6\left(\mathrm{CH}_{2}\right), 28.4\left(\mathrm{CH}_{3}\right)$. HRMS (ESI) $\mathrm{m} / \mathrm{z}$ : $[\mathrm{M}+\mathrm{H}]^{+}$Calcd for $\mathrm{C}_{18} \mathrm{H}_{24} \mathrm{~N}_{3} \mathrm{O}_{2}$ 314.1863; Found 314.1866 .

\section{1-(4-Chlorophenyl)-N-cyclohexyl-6-oxo-3,4,6, 7,8,8a-}

hexahydropyrrolo[1,2-a]pyrazine-8a-carboxamide (6c). White solid (Hexane/AcOEt, 2:1). Yield 332 mg, 89 \%. M. p. 188-190 ${ }^{\circ} \mathrm{C} .{ }^{1} \mathrm{H}$ NMR $\left(300 \mathrm{MHz}, \mathrm{CDCl}_{3}\right) \delta 7.68(\mathrm{~d}, J=8.7 \mathrm{~Hz}, 2 \mathrm{H}), 7.35$ $(\mathrm{d}, J=8.7 \mathrm{~Hz}, 2 \mathrm{H}), 5.84(\mathrm{~d}, J=8.2 \mathrm{~Hz}, 1 \mathrm{H}), 4.06-3.67(\mathrm{~m}, 4 \mathrm{H})$, $3.33-3.11(\mathrm{~m}, 1 \mathrm{H}), 2.95-2.65(\mathrm{~m}, 2 \mathrm{H}), 2.42-2.18(\mathrm{~m}, 1 \mathrm{H}), 2.10-$ $0.81(\mathrm{~m}, 11 \mathrm{H}) .{ }^{13} \mathrm{C}\left\{{ }^{1} \mathrm{H}\right\} \mathrm{NMR}\left(75 \mathrm{MHz}, \mathrm{CDCl}_{3}\right) \delta 173.4(\mathrm{Cq})$, $167.9(\mathrm{Cq}), 165.8(\mathrm{Cq}), 136.7(\mathrm{Cq}), 134.2(\mathrm{Cq}), 129.1\left(\mathrm{CH}_{\mathrm{Ar}}\right)$, $128.6\left(\mathrm{CH}_{\mathrm{Ar}}\right), 66.6(\mathrm{Cq}), 49.4(\mathrm{CH}), 47.5\left(\mathrm{CH}_{2}\right), 34.6\left(\mathrm{CH}_{2}\right)$, $32.9\left(\mathrm{CH}_{2}\right), 32.5\left(\mathrm{CH}_{2}\right), 30.6\left(\mathrm{CH}_{2}\right), 29.9\left(\mathrm{CH}_{2}\right), 25.2\left(\mathrm{CH}_{2}\right)$, $24.8\left(\mathrm{CH}_{2}\right), 24.7\left(\mathrm{CH}_{2}\right)$. HRMS (ESI) m/z: $[\mathrm{M}+\mathrm{H}]^{+}$Calcd for $\mathrm{C}_{20} \mathrm{H}_{25} \mathrm{ClN}_{3} \mathrm{O}_{2}$ 374.1630; Found 374.1632.

$\mathrm{N}$-Cyclohexyl-3,3,4,4-tetramethyl-6-oxo-1-phenyl3,4,6, 7,8,8a-hexahydropyrrolo[1,2-a]pyrazine-8acarboxamide (6d). Orange solid (Hexane/AcOEt, 3:1). Yield $284 \mathrm{mg}, 72 \%$. M. p. $181-182{ }^{\circ} \mathrm{C}$. ${ }^{1} \mathrm{H}$ NMR $\left(300 \mathrm{MHz}, \mathrm{CDCl}_{3}\right)$ $\delta$ 7.90-7.79 (m, 2H), 7.40-7.38 (m, 3H), $5.92(\mathrm{~d}, J=8.2 \mathrm{~Hz}$, $1 \mathrm{H}), 3.89-3.57(\mathrm{~m}, 1 \mathrm{H}), 2.65$ (ddd, $J=16.5,12.3,8.4 \mathrm{~Hz}, 1 \mathrm{H})$, $2.48(\mathrm{dd}, J=12.7,8.4 \mathrm{~Hz}, 1 \mathrm{H}), 2.24(\mathrm{dd}, J=16.5,9.3 \mathrm{~Hz}, 1 \mathrm{H})$, $2.01(\mathrm{td}, J=12.3,9.3 \mathrm{~Hz}, 1 \mathrm{H}), 1.86-1.77(\mathrm{~m}, 3 \mathrm{H}), 1.54(\mathrm{~s}, 3 \mathrm{H})$, $1.50(\mathrm{~s}, 3 \mathrm{H}), 1.39(\mathrm{~s}, 3 \mathrm{H}), 1.23(\mathrm{~s}, 3 \mathrm{H}), 1.63-1.02(\mathrm{~m}, 7 \mathrm{H})$. ${ }^{13} \mathrm{C}\left\{{ }^{1} \mathrm{H}\right\}$ NMR $\left(75 \mathrm{MHz}, \mathrm{CDCl}_{3}\right) \delta 176.1(\mathrm{Cq}), 170.7(\mathrm{Cq})$, $137.9(\mathrm{Cq}), 129.7\left(\mathrm{CH}_{\mathrm{Ar}}\right), 128.9\left(\mathrm{CH}_{\mathrm{Ar}}\right), 128.4\left(\mathrm{CH}_{\mathrm{Ar}}\right), 67.9$ $(\mathrm{Cq}), 61.9(\mathrm{Cq}), 59.9(\mathrm{Cq}), 48.7(\mathrm{CH}), 33.6\left(\mathrm{CH}_{2}\right), 32.7\left(\mathrm{CH}_{2}\right)$, $32.1\left(\mathrm{CH}_{2}\right), 31.1\left(\mathrm{CH}_{2}\right), 27.3\left(\mathrm{CH}_{3}\right), 25.3\left(\mathrm{CH}_{2}\right), 24.6\left(\mathrm{CH}_{2}\right)$, $23.7\left(\mathrm{CH}_{3}\right), 22.8\left(\mathrm{CH}_{3}\right), 21.8\left(\mathrm{CH}_{3}\right)$. HRMS (ESI) m/z: $[\mathrm{M}+$ $\mathrm{H}]^{+}$Calcd for $\mathrm{C}_{24} \mathrm{H}_{34} \mathrm{~N}_{3} \mathrm{O}_{2}$ 396.2646; Found 396.2658.

$\mathrm{N}$-(tert-Butyl)-3,3,4,4-tetramethyl-6-oxo-1-phenyl3,4,6,7,8,8a-hexahydropyrrolo[1,2-a]pyrazine-8acarboxamide (6e). Pale orange solid (Hexane/AcOEt, 5:1). Yield $214 \mathrm{mg}, 58 \%$. M. p. $148-150{ }^{\circ} \mathrm{C}$. ${ }^{1} \mathrm{H}$ NMR $(300 \mathrm{MHz}$, $\left.\mathrm{CDCl}_{3}\right) \delta 7.88-7.85(\mathrm{~m}, 2 \mathrm{H}), 7.42-7.40(\mathrm{~m}, 3 \mathrm{H}), 5.78(\mathrm{~s}, 1 \mathrm{H})$, 2.72-2.59 (m, 1H), 2.49 (dd, $J=12.5,8.4 \mathrm{~Hz}, 1 \mathrm{H}), 2.26(\mathrm{dd}, J$ $=16.5,9.5 \mathrm{~Hz}, 1 \mathrm{H}), 2.09-1.98(\mathrm{~m}, 1 \mathrm{H}), 1.52(\mathrm{~s}, 6 \mathrm{H}), 1.39(\mathrm{~s}$,
3H), 1.27 (s, 3H), $1.26(\mathrm{~s}, 9 \mathrm{H}) .{ }^{13} \mathrm{C}\left\{{ }^{1} \mathrm{H}\right\} \mathrm{NMR}\left(75 \mathrm{MHz}, \mathrm{CDCl}_{3}\right)$ $\delta 175.8(\mathrm{Cq}), 170.5(\mathrm{Cq}), 138.1(\mathrm{Cq}), 129.8\left(\mathrm{CH}_{\mathrm{Ar}}\right), 128.9$ $\left(\mathrm{CH}_{\mathrm{Ar}}\right), 128.5\left(\mathrm{CH}_{\mathrm{Ar}}\right), 61.9(\mathrm{Cq}), 59.8(\mathrm{Cq}), 51.8(\mathrm{Cq}), 33.5$ $\left(\mathrm{CH}_{2}\right), 31.2\left(\mathrm{CH}_{2}\right), 28.2\left(\mathrm{CH}_{3}\right), 27.2\left(\mathrm{CH}_{3}\right), 23.8\left(\mathrm{CH}_{3}\right), 22.8$ $\left(\mathrm{CH}_{3}\right), 21.8\left(\mathrm{CH}_{3}\right)$. HRMS (ESI) $\mathrm{m} / \mathrm{z}:[\mathrm{M}+\mathrm{H}]^{+}$Calcd for $\mathrm{C}_{22} \mathrm{H}_{32} \mathrm{~N}_{3} \mathrm{O}_{2}$ 370.2489; Found 370.2499 .

$\mathrm{N}$-Butyl-3,3,4,4-tetramethyl-6-oxo-1-phenyl-3,4,6,7,8,8ahexahydropyrrolo[1,2-a]pyrazine-8a-carboxamide

Orange solid (Hexane/AcOEt, 3:1). Yield $251 \mathrm{mg}, 68 \%$. M. p. 149-150 ${ }^{\circ} \mathrm{C} .{ }^{1} \mathrm{H}$ NMR $\left(300 \mathrm{MHz}, \mathrm{CDCl}_{3}\right) \delta 7.93-7.81(\mathrm{~m}, 2 \mathrm{H})$, 7.40-7.31 (m, 3H), $6.28(\mathrm{t}, J=5.7 \mathrm{~Hz}, 1 \mathrm{H}), 3.55-3.04(\mathrm{~m}, 2 \mathrm{H})$, 2.66 (ddd, $J=16.6,12.7,8.4 \mathrm{~Hz}, 1 \mathrm{H}), 2.46(\mathrm{dd}, J=12.7,8.7$ $\mathrm{Hz}, 1 \mathrm{H}), 2.22$ (dd, $J=16.6,9.9 \mathrm{~Hz}, 1 \mathrm{H}), 2.07-1.90$ (m, 1H), $1.55(\mathrm{~s}, 3 \mathrm{H}), 1.47(\mathrm{~s}, 3 \mathrm{H}), 1.45-1.40(\mathrm{~m}, 2 \mathrm{H}), 1.39(\mathrm{~s}, 3 \mathrm{H}), 1.31-$ $1.22(\mathrm{~m}, 2 \mathrm{H}), 1.20(\mathrm{~s}, 3 \mathrm{H}), 0.87(\mathrm{t}, J=7.3 \mathrm{~Hz}, 3 \mathrm{H}) .{ }^{13} \mathrm{C}\left\{{ }^{1} \mathrm{H}\right\}$ NMR $\left(75 \mathrm{MHz}, \mathrm{CDCl}_{3}\right) \delta 176.2(\mathrm{Cq}), 171.8(\mathrm{Cq}), 165.6(\mathrm{Cq})$, $138.0(\mathrm{Cq}), 129.6\left(\mathrm{CH}_{\mathrm{Ar}}\right), 128.9\left(\mathrm{CH}_{\mathrm{Ar}}\right), 128.3\left(\mathrm{CH}_{\mathrm{Ar}}\right), 67.9$ $(\mathrm{Cq}), 61.8(\mathrm{Cq}), 60.0(\mathrm{Cq}), 39.9\left(\mathrm{CH}_{2}\right), 33.5\left(\mathrm{CH}_{2}\right), 31.1\left(\mathrm{CH}_{2}\right)$, $30.9\left(\mathrm{CH}_{2}\right), 27.4\left(\mathrm{CH}_{3}\right), 23.5\left(\mathrm{CH}_{3}\right), 22.8\left(\mathrm{CH}_{3}\right), 21.8\left(\mathrm{CH}_{3}\right)$, $20.1\left(\mathrm{CH}_{2}\right), 13.6\left(\mathrm{CH}_{3}\right)$. HRMS (ESI) m/z: $[\mathrm{M}+\mathrm{H}]^{+}$Calcd for $\mathrm{C}_{22} \mathrm{H}_{32} \mathrm{~N}_{3} \mathrm{O}_{2}$ 370.2489; Found 370.2498.

(3aR,5aS,9aS)-N-Cyclohexyl-1-oxo-4-phenyl1,2,3,3a,5a,6, 7,8,9,9a-decahydropyrrolo[1,2-a]quinoxaline3a-carboxamide (7a). Brown solid. (Hexane/AcOEt, 2:1). Yield $350 \mathrm{mg}, 89 \%$. M. p. $158-160^{\circ} \mathrm{C}$. $[\alpha]_{\mathrm{D}}=+148.9^{\circ}(\mathrm{c}=1.41$, $\left.\mathrm{CH}_{3} \mathrm{OH}\right) .{ }^{1} \mathrm{H}$ NMR $\left(300 \mathrm{MHz}, \mathrm{CDCl}_{3}\right) \delta: 7.69(\mathrm{~d}, J=7.4 \mathrm{~Hz}$, 2H), 7.44-7.34 (m, 3H), $5.56(\mathrm{~d}, J=8.4 \mathrm{~Hz}, 1 \mathrm{H}), 3.90-3.81(\mathrm{~m}$, $1 \mathrm{H}), 3.41$ (ddd, $J=12.2,9.8,3.7 \mathrm{~Hz}, 1 \mathrm{H}), 3.14(\mathrm{ddd}, J=13.0$, 9.8, 3.6 Hz, 1H), 2.92-2.64 (m, 4H), 2.46-2.23 (m, 4H), 1.94$0.93(\mathrm{~m}, 14 \mathrm{H}) .{ }^{13} \mathrm{C}\left\{{ }^{1} \mathrm{H}\right\} \mathrm{NMR}\left(75 \mathrm{MHz}, \mathrm{CDCl}_{3}\right) \delta: 173.7(\mathrm{Cq})$, $168.5(\mathrm{Cq}), 163.5(\mathrm{Cq}), 136.0(\mathrm{Cq}), 130.6\left(\mathrm{CH}_{\mathrm{Ar}}\right), 128.5\left(\mathrm{CH}_{\mathrm{Ar}}\right)$, $127.9\left(\mathrm{CH}_{\mathrm{Ar}}\right), 70.3(\mathrm{Cq}), 63.1(\mathrm{CH}), 57.3(\mathrm{CH}), 49.1(\mathrm{CH}), 33.8$ $\left(\mathrm{CH}_{2}\right), 33.0\left(\mathrm{CH}_{2}\right), 32.3\left(\mathrm{CH}_{2}\right), 29.2\left(\mathrm{CH}_{2}\right), 28.1\left(\mathrm{CH}_{2}\right), 26.0$ $\left(\mathrm{CH}_{2}\right), 25.5\left(\mathrm{CH}_{2}\right), 25.2\left(\mathrm{CH}_{2}\right), 24.6\left(\mathrm{CH}_{2}\right), 24.4\left(\mathrm{CH}_{2}\right)$. HRMS (ESI) $\mathrm{m} / \mathrm{z}$ : $[\mathrm{M}+\mathrm{H}]^{+}$Calcd for $\mathrm{C}_{24} \mathrm{H}_{32} \mathrm{~N}_{3} \mathrm{O}_{2}$ 394.2489; Found 394.2492 .

(3aR, 5aS,9aS)-N-Cyclohexyl-1-oxo-4-(p-tolyl)1,2,3,3a,5a, 6, 7,8,9,9a-decahydropyrrolo [1,2-a]quinoxaline3a-carboxamide (7b). Yellow solid. (Hexane/AcOEt, 2:1). Yield $370 \mathrm{mg}, 91 \%$. M. p. $69-71^{\circ} \mathrm{C}$. $[\alpha]_{\mathrm{D}}=+123.6^{\circ}(\mathrm{c}=1.11$, $\left.\mathrm{CH}_{3} \mathrm{OH}\right) .{ }^{1} \mathrm{H}$ NMR $\left(300 \mathrm{MHz}, \mathrm{CDCl}_{3}\right) \delta: 7.55(\mathrm{~d}, J=8.1 \mathrm{~Hz}$, $2 \mathrm{H}), 7.14(\mathrm{~d}, J=8.1 \mathrm{~Hz}, 2 \mathrm{H}), 5.55(\mathrm{~d}, J=8.2 \mathrm{~Hz}, 1 \mathrm{H}), 3.87-$ $3.77(\mathrm{~m}, 1 \mathrm{H}), 3.36$ (ddd, $J=12.7,9.9,3.7 \mathrm{~Hz}, 1 \mathrm{H}), 3.09$ (ddd, $J=13.0,9.9,3.5,1 \mathrm{H}), 2.89-2.60(\mathrm{~m}, 4 \mathrm{H}), 2.33$ (s, 3H), 2.43$2.18(\mathrm{~m}, 4 \mathrm{H}), 1.92-0.87(\mathrm{~m}, 14 \mathrm{H}) .{ }^{13} \mathrm{C}\left\{{ }^{1} \mathrm{H}\right\} \mathrm{NMR}(75 \mathrm{MHz}$, $\left.\mathrm{CDCl}_{3}\right) \delta: 173.6(\mathrm{Cq}), 168.6(\mathrm{Cq}), 163.1(\mathrm{Cq}), 140.7(\mathrm{Cq}), 132.9$ $(\mathrm{Cq}), 129.1\left(\mathrm{CH}_{\mathrm{Ar}}\right), 127.8\left(\mathrm{CH}_{\mathrm{Ar}}\right), 70.2(\mathrm{Cq}), 63.0(\mathrm{CH}), 57.2$ $(\mathrm{CH}), 49.0(\mathrm{CH}), 33.8\left(\mathrm{CH}_{2}\right), 32.8\left(\mathrm{CH}_{2}\right), 32.2\left(\mathrm{CH}_{2}\right), 32.1$ $\left(\mathrm{CH}_{2}\right), 29.2\left(\mathrm{CH}_{2}\right), 28.1\left(\mathrm{CH}_{2}\right), 26.0\left(\mathrm{CH}_{2}\right), 25.5\left(\mathrm{CH}_{2}\right), 25.2$ $\left(\mathrm{CH}_{2}\right), 24.6\left(\mathrm{CH}_{2}\right), 24.4\left(\mathrm{CH}_{2}\right), 21.2\left(\mathrm{CH}_{3}\right)$. HRMS (ESI) m/z: $[\mathrm{M}+\mathrm{H}]^{+}$Calcd for $\mathrm{C}_{25} \mathrm{H}_{34} \mathrm{~N}_{3} \mathrm{O}_{2}$ 408.2646; Found 408.2648.

(3aR,5aS,9aS)-N-(tert-Butyl)-4-phenyl-1-oxo1,2,3,3a,5a,6, 7,8,9,9a-decahydropyrrolo[1,2-a]quinoxaline$3 a$-carboxamide (7c). Yellow sticky solid. (Hexane/AcOEt, 2:1). Yield $316 \mathrm{mg}, 86 \%$. $[\alpha]_{\mathrm{D}}=+161.4^{\circ}\left(\mathrm{c}=0.95, \mathrm{CH}_{3} \mathrm{OH}\right)$. ${ }^{1} \mathrm{H}$ NMR $\left(300 \mathrm{MHz}, \mathrm{CDCl}_{3}\right) \delta: 7.69-7.65(\mathrm{~m}, 2 \mathrm{H}), 7.42-7.33$ $(\mathrm{m}, 3 \mathrm{H}), 5.40(\mathrm{~s}, 1 \mathrm{H}), 3.39(\mathrm{ddd}, J=12.6,9.5,3.5 \mathrm{~Hz}, 1 \mathrm{H}), 3.14$ (ddd, $J=12.7,9.5,3.4 \mathrm{~Hz}, 1 \mathrm{H}), 2.87-2.63$ (m, 3H), 2.45-2.20 (m, 3H), 1.94-1.78 (m, 3H), 1.52-1.17 (m, 3H), 1.27 (s, 9H). ${ }^{13} \mathrm{C}\left\{{ }^{1} \mathrm{H}\right\}$ NMR $\left(75 \mathrm{MHz}, \mathrm{CDCl}_{3}\right) \delta: 173.7(\mathrm{Cq}), 168.6(\mathrm{Cq})$, $163.6(\mathrm{Cq}), 135.8(\mathrm{Cq}), 130.5\left(\mathrm{CH}_{\mathrm{Ar}}\right), 128.6\left(\mathrm{CH}_{\mathrm{Ar}}\right), 127.9$ $\left(\mathrm{CH}_{\mathrm{Ar}}\right), 71.0(\mathrm{Cq}), 63.2(\mathrm{CH}), 57.3(\mathrm{CH}), 52.5(\mathrm{Cq}), 33.9\left(\mathrm{CH}_{2}\right)$, $32.3\left(\mathrm{CH}_{2}\right), 29.2\left(\mathrm{CH}_{2}\right), 28.5\left(\mathrm{CH}_{3}\right), 28.2\left(\mathrm{CH}_{2}\right), 26.2\left(\mathrm{CH}_{2}\right)$, 
25.6 $\left(\mathrm{CH}_{2}\right)$. HRMS (ESI) m/z: $[\mathrm{M}+\mathrm{H}]^{+}$Calcd for $\mathrm{C}_{22} \mathrm{H}_{30} \mathrm{~N}_{3} \mathrm{O}_{2}$ 368.2333; Found 368.2345.

(3S,4S,8aS)-N-Cyclohexyl-6-oxo-1,3,4-triphenyl-3,4,6,7,8,8ahexahydropyrrolo[1,2-a]pyrazine-8a-carboxamide (7d). Yellow solid (Hexane/AcOEt, 3:1). Yield $452 \mathrm{mg}, 92 \%$. M. p. $60-62^{\circ} \mathrm{C} .[\alpha]_{\mathrm{D}}=-95.4^{\circ}\left(\mathrm{c}=0.49, \mathrm{CH}_{3} \mathrm{OH}\right) .{ }^{1} \mathrm{H}$ NMR $(300 \mathrm{MHz}$, $\left.\mathrm{CDCl}_{3}\right)$ 8: 7.85-7.81 (m, 2H), 7.48-7.42 (m, 3H) 7.27-7.21 (m, $8 \mathrm{H}), 7.11-7.07(\mathrm{~m}, 2 \mathrm{H}), 5.74(\mathrm{~d}, J=7.9 \mathrm{~Hz}, 1 \mathrm{H}), 4.94(\mathrm{~d}, J=$ $8.6 \mathrm{~Hz}, 1 \mathrm{H}), 4.80(\mathrm{~d}, J=8.6 \mathrm{~Hz}, 1 \mathrm{H}), 3.82-3.70(\mathrm{~m}, 1 \mathrm{H}), 2.98-$ $2.70(\mathrm{~m}, 2 \mathrm{H}), 2.38-2.19(\mathrm{~m}, 2 \mathrm{H}), 1.92-0.82(\mathrm{~m}, 10 \mathrm{H}) .{ }^{13} \mathrm{C}\left\{{ }^{1} \mathrm{H}\right\}$ NMR (75 MHz, $\left.\mathrm{CDCl}_{3}\right) \delta: 175.3(\mathrm{Cq}), 171.6(\mathrm{Cq}), 169.5(\mathrm{Cq})$, $140.1(\mathrm{Cq}), 138.3(\mathrm{Cq}), 136.5(\mathrm{Cq}), 130.5\left(\mathrm{CH}_{\mathrm{Ar}}\right), 128.6\left(\mathrm{CH}_{\mathrm{Ar}}\right)$, $128.4\left(\mathrm{CH}_{\mathrm{Ar}}\right), 128.3\left(\mathrm{CH}_{\mathrm{Ar}}\right), 128.0\left(\mathrm{CH}_{\mathrm{Ar}}\right), 128.0\left(\mathrm{CH}_{\mathrm{Ar}}\right), 127.9$ $\left(\mathrm{CH}_{\mathrm{Ar}}\right), 127.7\left(\mathrm{CH}_{\mathrm{Ar}}\right), 127.6\left(\mathrm{CH}_{\mathrm{Ar}}\right), 67.3(\mathrm{Cq}), 65.7(\mathrm{CH}), 59.7$ (CH), $49.2(\mathrm{CH}), 32.5\left(\mathrm{CH}_{2}\right), 32.4\left(\mathrm{CH}_{2}\right), 32.3\left(\mathrm{CH}_{2}\right), 30.2$ $\left(\mathrm{CH}_{2}\right), 29.7\left(\mathrm{CH}_{2}\right), 25.2\left(\mathrm{CH}_{2}\right), 24.7\left(\mathrm{CH}_{2}\right), 24.6\left(\mathrm{CH}_{2}\right)$. HRMS (ESI) $\mathrm{m} / \mathrm{z}$ : $[\mathrm{M}+\mathrm{H}]^{+}$Calcd for $\mathrm{C}_{32} \mathrm{H}_{34} \mathrm{~N}_{3} \mathrm{O}_{2}$ 492.2646; Found 492.2653.

(3S,4S,8aS)-N-(tert-Butyl)-6-oxo-1,3,4-triphenyl-3,4,6, 7,8,8ahexahydropyrrolo[1,2-a]pyrazine-8a-carboxamide (7e). Red solid. (Hexane/AcOEt, 4:1). Yield $404 \mathrm{mg}, 87$ \%. M. p. 56-58 ${ }^{\circ} \mathrm{C} .[\alpha]_{\mathrm{D}}=-105.4^{\circ}\left(\mathrm{c}=0.57, \mathrm{CH}_{3} \mathrm{OH}\right) .{ }^{1} \mathrm{H}$ NMR $(300 \mathrm{MHz}$, $\left.\mathrm{CDCl}_{3}\right)$ \&: 7.87-7.83 (m, 2H), 7.48-7.45 (m, 3H), 7.33-7.23 (m, $8 \mathrm{H}), 7.14-7.10(\mathrm{~m}, 2 \mathrm{H}), 5.60(\mathrm{~s}, 1 \mathrm{H}), 5.02(\mathrm{~d}, J=8.3 \mathrm{~Hz}, 1 \mathrm{H})$, $4.88(\mathrm{~d}, J=8.3 \mathrm{~Hz}, 1 \mathrm{H}), 2.92-2.64(\mathrm{~m}, 2 \mathrm{H}), 2.40-2.18(\mathrm{~m}, 2 \mathrm{H})$, $1.23(\mathrm{~s}, 9 \mathrm{H}) .{ }^{13} \mathrm{C}\left\{{ }^{1} \mathrm{H}\right\}$ NMR $\left(75 \mathrm{MHz}, \mathrm{CDCl}_{3}\right) \delta: 175.5(\mathrm{Cq})$, $171.4(\mathrm{Cq}), 169.4(\mathrm{Cq}), 140.0(\mathrm{Cq}), 138.5(\mathrm{Cq}), 136.6(\mathrm{Cq})$, $130.5\left(\mathrm{CH}_{\mathrm{Ar}}\right), 128.5\left(\mathrm{CH}_{\mathrm{Ar}}\right), 128.5\left(\mathrm{CH}_{\mathrm{Ar}}\right), 128.3\left(\mathrm{CH}_{\mathrm{Ar}}\right), 128.1$ $\left(\mathrm{CH}_{\mathrm{Ar}}\right), 128.0\left(\mathrm{CH}_{\mathrm{Ar}}\right), 127.9\left(\mathrm{CH}_{\mathrm{Ar}}\right), 127.7\left(\mathrm{CH}_{\mathrm{Ar}}\right), 127.6$ $\left(\mathrm{CH}_{\mathrm{Ar}}\right), 67.8(\mathrm{Cq}), 65.1(\mathrm{CH}), 59.2(\mathrm{CH}), 52.1(\mathrm{Cq}), 32.3\left(\mathrm{CH}_{2}\right)$, $30.2\left(\mathrm{CH}_{2}\right), 28.2\left(\mathrm{CH}_{3}\right)$. HRMS (ESI) m/z: $[\mathrm{M}+\mathrm{H}]^{+}$Calcd for $\mathrm{C}_{30} \mathrm{H}_{32} \mathrm{~N}_{3} \mathrm{O}_{2}$ 466.2489; Found 466.2496

(3S,4S,8aS)-N-Butyl-6-oxo-1,3,4-triphenyl-3,4,6,7,8,8ahexahydropyrrolo[1,2-a]pyrazine-8a-carboxamide Yellow solid. (Hexane/AcOEt, 5:1). Yield $274 \mathrm{mg}, 59 \%$. M. p. $141-143{ }^{\circ} \mathrm{C} .[\alpha]_{\mathrm{D}}=-51.4^{\circ}\left(\mathrm{c}=0.44, \mathrm{CH}_{3} \mathrm{OH}\right) .{ }^{1} \mathrm{H}$ NMR $(300$ $\left.\mathrm{MHz}, \mathrm{CDCl}_{3}\right) \delta 7.90-7.78(\mathrm{~m}, 2 \mathrm{H}), 7.50-7.38(\mathrm{~m}, 3 \mathrm{H}), 7.36-$ $7.22(\mathrm{~m}, 8 \mathrm{H}), 7.18-7.05(\mathrm{~m}, 2 \mathrm{H}), 5.62(\mathrm{t}, J=5.7 \mathrm{~Hz}, 1 \mathrm{H}), 5.21$ $(\mathrm{d}, J=7.1 \mathrm{~Hz}, 1 \mathrm{H}), 4.98(\mathrm{~d}, J=7.1 \mathrm{~Hz}, 1 \mathrm{H}), 3.14(\mathrm{td}, J=13.2$, $7.1 \mathrm{~Hz}, 1 \mathrm{H}), 2.98-2.78(\mathrm{~m}, 3 \mathrm{H}), 2.30-2.20(\mathrm{~m}, 2 \mathrm{H}), 1.36-1.04$ $(\mathrm{m}, 4 \mathrm{H}), 0.83(\mathrm{t}, J=7.2 \mathrm{~Hz}, 3 \mathrm{H}) .{ }^{13} \mathrm{C}\left\{{ }^{1} \mathrm{H}\right\}$ NMR $(75 \mathrm{MHz}$, $\left.\mathrm{CDCl}_{3}\right) \delta 175.1(\mathrm{Cq}), 170.1(\mathrm{Cq}), 140.0(\mathrm{Cq}), 138.3(\mathrm{Cq}), 136.1$ $(\mathrm{Cq}), 130.7\left(\mathrm{CH}_{\mathrm{Ar}}\right), 128.6\left(\mathrm{CH}_{\mathrm{Ar}}\right), 128.5\left(\mathrm{CH}_{\mathrm{Ar}}\right), 128.4\left(\mathrm{CH}_{\mathrm{Ar}}\right)$, $128.0\left(\mathrm{CH}_{\mathrm{Ar}}\right), 127.9\left(\mathrm{CH}_{\mathrm{Ar}}\right), 127.8\left(\mathrm{CH}_{\mathrm{Ar}}\right), 127.7\left(\mathrm{CH}_{\mathrm{Ar}}\right), 127.6$ $\left(\mathrm{CH}_{\mathrm{Ar}}\right), 66.7(\mathrm{Cq}), 64.5(\mathrm{CH}), 58.5(\mathrm{CH}), 39.9\left(\mathrm{CH}_{2}\right), 32.1$ $\left(\mathrm{CH}_{2}\right), 30.9\left(\mathrm{CH}_{2}\right), 30.3\left(\mathrm{CH}_{2}\right), 20.0\left(\mathrm{CH}_{2}\right), 13.6\left(\mathrm{CH}_{3}\right)$. HRMS (ESI) $\mathrm{m} / \mathrm{z}$ : $[\mathrm{M}+\mathrm{H}]^{+}$Calcd for $\mathrm{C}_{30} \mathrm{H}_{32} \mathrm{~N}_{3} \mathrm{O}_{2}$ 466.2489; Found 466.2504 .

$\left(3 a R^{*}, 5 a S^{*}, 9 a R^{*}\right)-N$-Cyclohexyl-1-oxo-4-phenyl1,2,3,3a,5a,6,7,8,9,9a-decahydropyrrolo[1,2-a]quinoxaline3a-carboxamide (7g). Pale yellow solid. (Hexane/AcOEt, 3:1). Yield $365 \mathrm{mg}, 93 \%$. M. p. $156-158^{\circ} \mathrm{C}$. ${ }^{1} \mathrm{H}$ NMR $(300 \mathrm{MHz}$, $\left.\mathrm{CDCl}_{3}\right) \delta: 7.75(\mathrm{dd}, \mathrm{J}=7.6,2.0 \mathrm{~Hz}, 2 \mathrm{H}), 7.47-7.38(\mathrm{~m}, 3 \mathrm{H})$, $5.61(\mathrm{~d}, \mathrm{~J}=8.2 \mathrm{~Hz}, 1 \mathrm{H}), 4.43-4.25(\mathrm{~m}, 1 \mathrm{H}), 3.72-3.39(\mathrm{~m}, 2 \mathrm{H})$, 3.00-2.77 (m, 2H), 2.48-2.31 (m, 3H), 1.88-0.81 (m, 17H). ${ }^{13} \mathrm{C}\left\{{ }^{1} \mathrm{H}\right\}$ NMR $\left(75 \mathrm{MHz}, \mathrm{CDCl}_{3}\right) \delta: 174.8(\mathrm{Cq}), 169.2(\mathrm{Cq})$, $163.9(\mathrm{Cq}), 138.3(\mathrm{Cq}), 130.1\left(\mathrm{CH}_{\mathrm{Ar}}\right), 128.4\left(\mathrm{CH}_{\mathrm{Ar}}\right), 128.0$ $\left(\mathrm{CH}_{\mathrm{Ar}}\right), 66.3(\mathrm{Cq}), 56.6(\mathrm{CH}), 48.6(\mathrm{CH}), 46.7(\mathrm{CH}), 33.8\left(\mathrm{CH}_{2}\right)$, $32.4\left(\mathrm{CH}_{2}\right), 32.3\left(\mathrm{CH}_{2}\right), 32.2\left(\mathrm{CH}_{2}\right), 31.2\left(\mathrm{CH}_{2}\right), 25.8\left(\mathrm{CH}_{2}\right)$, $25.3\left(\mathrm{CH}_{2}\right), 24.6\left(\mathrm{CH}_{2}\right), 24.4\left(\mathrm{CH}_{2}\right), 20.7\left(\mathrm{CH}_{2}\right)$. HRMS (ESI) $\mathrm{m} / \mathrm{z}:[\mathrm{M}+\mathrm{Na}]^{+}$Calcd for $\mathrm{C}_{24} \mathrm{H}_{31} \mathrm{NaN}_{3} \mathrm{O}_{2}$ 416.2308; Found 416.2312 . $\left(3 a R^{*}, 5 a S^{*}, 9 a R^{*}\right)-N$-(tert-Butyl)-1-oxo-4-phenyl1,2,3,3a,5a,6,7,8,9,9a-decahydropyrrolo[1,2-a]quinoxaline3a-carboxamide (7h). White solid. (Hexane/AcOEt, 3:1). Yield $315 \mathrm{mg}, 86 \%$. M. p. $102-104{ }^{\circ} \mathrm{C} .{ }^{1} \mathrm{H}$ NMR $(300 \mathrm{MHz}$, $\left.\mathrm{CDCl}_{3}\right)$ 8: 7.76-7.73 (m, 2H), 7.46-7.40 (m, 3H), $5.49(\mathrm{~s}, 1 \mathrm{H})$, 4.38-4.30 (m, 1H), 3.72-3.68 (m, 1H), 2.97-2.81 (m, 2H), 2.46$2.23(\mathrm{~m}, 3 \mathrm{H}), 1.88-0.96(\mathrm{~m}, 7 \mathrm{H}), 1.12(\mathrm{~s}, 9 \mathrm{H}) .{ }^{13} \mathrm{C}\left\{{ }^{1} \mathrm{H}\right\} \mathrm{NMR}$ $\left(75 \mathrm{MHz}, \mathrm{CDCl}_{3}\right) \delta: 174.6(\mathrm{Cq}), 168.9(\mathrm{Cq}), 163.7(\mathrm{Cq}), 137.8$ $(\mathrm{Cq}), 130.1\left(\mathrm{CH}_{\mathrm{Ar}}\right), 128.4\left(\mathrm{CH}_{\mathrm{Ar}}\right), 127.8\left(\mathrm{CH}_{\mathrm{Ar}}\right), 66.7(\mathrm{Cq}), 56.5$ $(\mathrm{CH}), 51.5(\mathrm{Cq}), 46.5(\mathrm{CH}), 33.5\left(\mathrm{CH}_{2}\right), 32.3\left(\mathrm{CH}_{2}\right), 31.0\left(\mathrm{CH}_{2}\right)$, $21.9\left(\mathrm{CH}_{3}\right), 25.7\left(\mathrm{CH}_{2}\right), 24.5\left(\mathrm{CH}_{2}\right), 20.6\left(\mathrm{CH}_{2}\right)$. HRMS (ESI) $\mathrm{m} / \mathrm{z}:[\mathrm{M}+\mathrm{Na}]^{+}$Calcd for $\mathrm{C}_{22} \mathrm{H}_{29} \mathrm{NaN}_{3} \mathrm{O}_{2}$ 390.2152; Found 390.2156 .

$\left(3 R^{*}, 4 S^{*}, 8 a S^{*}\right)-N$-Cyclohexyl-6-oxo-1,3,4-triphenyl3,4,6,7,8,8a-hexahydropyrrolo[1,2-a]pyrazine-8a-

carboxamide (7i). Orange solid. (Hexane/AcOEt, 3:1). Yield $457 \mathrm{mg}, 93 \%$. M. p. $144-145{ }^{\circ} \mathrm{C} .{ }^{1} \mathrm{H}$ NMR $\left(300 \mathrm{MHz}, \mathrm{CDCl}_{3}\right)$ $\delta$ 7.94-7.91 (m, 2H), 7.78-7.75 (m, 2H), 7.45-7.12 (m, 11H), $6.28(\mathrm{~d}, J=5.5 \mathrm{~Hz}, 1 \mathrm{H}), 5.15(\mathrm{~d}, J=5.5 \mathrm{~Hz}, 1 \mathrm{H}), 4.45(\mathrm{~d}, J=$ $7.7 \mathrm{~Hz}, 1 \mathrm{H}), 3.43(\mathrm{dd}, J=11.9,7.8 \mathrm{~Hz}, 1 \mathrm{H}), 3.09-2.93(\mathrm{~m}, 2 \mathrm{H})$, $2.53(\mathrm{dd}, J=16.5,8.8 \mathrm{~Hz}, 1 \mathrm{H}), 2.28(\mathrm{td}, J=11.9,8.8 \mathrm{~Hz}, 1 \mathrm{H})$, 1.37-0.76 (m, 8H), 0.21-0.07 (m, 2H). ${ }^{13} \mathrm{C}\left\{{ }^{1} \mathrm{H}\right\}$ NMR $(75 \mathrm{MHz}$, $\left.\mathrm{CDCl}_{3}\right) \delta 176.2(\mathrm{Cq}), 167.2(\mathrm{Cq}), 163.5(\mathrm{Cq}), 140.0(\mathrm{Cq}), 137.1$ $(\mathrm{Cq}), 135.1(\mathrm{Cq}), 130.5\left(\mathrm{CH}_{\mathrm{Ar}}\right), 130.4\left(\mathrm{CH}_{\mathrm{Ar}}\right), 128.9\left(\mathrm{CH}_{\mathrm{Ar}}\right)$, $128.5\left(\mathrm{CH}_{\mathrm{Ar}}\right), 128.3\left(\mathrm{CH}_{\mathrm{Ar}}\right), 128.0\left(\mathrm{CH}_{\mathrm{Ar}}\right), 127.8\left(\mathrm{CH}_{\mathrm{Ar}}\right), 126.8$ $\left(\mathrm{CH}_{\mathrm{Ar}}\right), 126.4\left(\mathrm{CH}_{\mathrm{Ar}}\right), 67.2(\mathrm{Cq}), 61.3(\mathrm{CH}), 52.4(\mathrm{CH}), 48.5$ $(\mathrm{CH}), 34.3\left(\mathrm{CH}_{2}\right), 31.5\left(\mathrm{CH}_{2}\right), 31.2\left(\mathrm{CH}_{2}\right), 25.2\left(\mathrm{CH}_{2}\right), 24.5$ $\left(\mathrm{CH}_{2}\right), 24.4\left(\mathrm{CH}_{2}\right)$. HRMS (ESI) $\mathrm{m} / \mathrm{z}:[\mathrm{M}+\mathrm{H}]^{+}$Calcd for $\mathrm{C}_{32} \mathrm{H}_{34} \mathrm{~N}_{3} \mathrm{O}_{2}$ 492.2646; Found 492.2655 .

$\left(3 R^{*}, 4 S^{*}, 8 a S^{*}\right)$ - $N$-(tert-Butyl)-6-oxo-1,3,4-triphenyl3,4,6,7,8,8a-hexahydropyrrolo[1,2-a]pyrazine-8a-

carboxamide (7j). Pale yellow solid. (Hexane/AcOEt, 3:1). Yield 446 mg, 96 \%. M. p. $194-195{ }^{\circ} \mathrm{C} .{ }^{1} \mathrm{H}$ NMR $(300 \mathrm{MHz}$, $\left.\mathrm{CDCl}_{3}\right) \delta$ 7.96-7.93 (m, 2H), 7.80-7.77 (m, 2H), 7.46-7.12 (m, $11 \mathrm{H}), 6.30(\mathrm{~d}, J=5.5 \mathrm{~Hz}, 1 \mathrm{H}), 5.15(\mathrm{~d}, J=5.5 \mathrm{~Hz}, 1 \mathrm{H}), 4.37(\mathrm{~s}$, $1 \mathrm{H}, \mathrm{NH}), 3.39$ (dd, $J=11.9,8.0 \mathrm{~Hz}, 1 \mathrm{H}), 3.11$ (ddd, $J=16.5$, $11.9,8.0 \mathrm{~Hz}, 1 \mathrm{H}), 2.54(\mathrm{dd}, J=16.5,9.0 \mathrm{~Hz}, 1 \mathrm{H}), 2.24(\mathrm{td}, J=$ $11.9,9.0 \mathrm{~Hz}, 1 \mathrm{H}), 0.60(\mathrm{~s}, 9 \mathrm{H}) .{ }^{13} \mathrm{C}\left\{{ }^{1} \mathrm{H}\right\}$ NMR $(75 \mathrm{MHz}$, $\left.\mathrm{CDCl}_{3}\right) \delta 176.4(\mathrm{Cq}), 167.3(\mathrm{Cq}), 163.5(\mathrm{Cq}), 140.1(\mathrm{Cq}), 136.9$ $(\mathrm{Cq}), 135.2(\mathrm{Cq}), 130.5\left(\mathrm{CH}_{\mathrm{Ar}}\right), 128.9\left(\mathrm{CH}_{\mathrm{Ar}}\right), 128.5\left(\mathrm{CH}_{\mathrm{Ar}}\right)$, $128.3\left(\mathrm{CH}_{\mathrm{Ar}}\right), 127.9\left(\mathrm{CH}_{\mathrm{Ar}}\right), 127.8\left(\mathrm{CH}_{\mathrm{Ar}}\right), 126.8\left(\mathrm{CH}_{\mathrm{Ar}}\right), 126.3$ $\left(\mathrm{CH}_{\mathrm{Ar}}\right), 67.4(\mathrm{Cq}), 61.3(\mathrm{CH}), 52.2(\mathrm{CH}), 51.0(\mathrm{Cq}), 34.3\left(\mathrm{CH}_{2}\right)$, $31.7\left(\mathrm{CH}_{2}\right), 27.3\left(\mathrm{CH}_{3}\right)$. HRMS (ESI) m/z: $[\mathrm{M}+\mathrm{H}]^{+}$Calcd for $\mathrm{C}_{30} \mathrm{H}_{32} \mathrm{~N}_{3} \mathrm{O}_{2}$ 466.2489; Found 466.2498.

Computational studies. The geometries of all species were fully optimized at the B3LYP/6-31G** level. The nature of all optimized structures was determined using harmonic frequency analysis as true minima with no imaginary frequencies. All calculations were performed using the Gaussian 09 program. ${ }^{24}$

\section{ASSOCIATED CONTENT}

\section{Supporting Information}

The Supporting Information is available free of charge on the ACS Publications website.

Copies of ${ }^{1} \mathrm{H}$ NMR, ${ }^{13} \mathrm{C}$ NMR and high-resolution mass spectra, and computational data (PDF).

X-ray chrystallographic data for compounds $7 \mathbf{a}$ and $7 \mathrm{~g}$ (CIF). CCDC 1982178-1982179

\section{AUTHOR INFORMATION}




\section{Corresponding Author}

María García-Valverde- Departamento de Química, Facultad de Ciencias, Universidad de Burgos, Burgos 09001, Spain; orcid.org/0000-0002-3990-8388; Email: magaval@ubu.es

\section{Authors}

Pablo Pertejo-Departamento de Química, Facultad de Ciencias, Universidad de Burgos, Burgos 09001, Spain.

Beatriz González Saiz-Departamento de Química, Facultad de Ciencias, Universidad de Burgos, Burgos 09001, Spain. Roberto Quesada-Departamento de Química, Facultad de Ciencias, Universidad de Burgos, Burgos 09001, Spain; orcid.org/0000-0002-4835-8752

\section{Author Contributions}

The manuscript was written through contributions of all authors. All authors have given approval to the final version of the manuscript.

Notes

The authors declare no competing financial interest.

\section{ACKNOWLEDGMENT}

Funding from Consejería de Educación de la Junta de Castilla y León (project BU075G19) is gratefully acknowledged.

\section{REFERENCES}

1. Rathi, A. K.; Syed, R.; Shin, H.-S.; Patel, R. V. Piperazine derivatives for therapeutic use: a patent review (2010-present). Exp. Opin. Ther. Pat. 2016, 26, 777-797.

2. (a) Kumar, K. S.; Kumar, N. P.; Rajesham, B.; Kishan, G.; Akula, S.; Kancha, R. K. Silver-catalyzed synthesis of pyrrolopiperazine fused with oxazine/imidazole via a domino approach: evaluation of anti-cancer activity, New J. Chem. 2018, 42, 34-38. (b) Shaquiquzzaman, M.; Verma, G.; Marella, A.; Akhter, M.; Akhtar, W.; Khan, M. F.; Tasneem, S.; Alam. M. M. Piperazine scaffold: A remarkable tool in generation of diverse pharmacological agents. Eur. J. Med. Chem. 2015, 102, 487-529.

3. Borlinghaus, N.; Gergel, S.; Nestl, B. M. Biocatalytic access to piperazines from diamines and dicarbonyls. ACS Catal. 2018, 8, 3727-3732.

4. (a) Marichev, K. O.; Takacs, J. M. Ruthenium-catalyzed amination of secondary alcohols using borrowing hydrogen methodology. ACS Catal. 2016, 6, 2205-2210. (b) Nordstrøm, L. U.; Madsen, R. Iridium catalysed synthesis of piperazines from diols. Chem. Commun. 2007, 5034-5036. (c) Jenner, G.; Bitsi, G. Ruthenium-catalyzed synthesis of piperazines J. Mol. Cat. 1988, 45, 165-168.

5. Vairaprakash, P.; Periasamy, M. A simple method of synthesis of ( \pm -2,3-diarylpiperazines and a novel method of resolution of ( \pm )-2,3-diphenylpiperazine. J. Org. Chem. 2006, 71, 36363638 .

6. Ye, Z.; Adhikari, S.; Xia, Y.; Dai, M. Expedient syntheses of $\mathrm{N}$-heterocycles via intermolecular amphoteric diamination of allenes. Nat. Commun. 2018, 9, 721 .

7. (a) Du, H.; Rodriguez, J.; Bugaut, X.; Constantieux, T. Organocatalytic enantioselective multicomponent synthesis of pyrrolopiperazines. Adv. Synth. Catal. 2014, 356, 851-856. (b) Liéby-Muller, F.; Constantieux, T.; Rodriguez, J. Highly efficient access to original polycyclic pyrrolopiperazine scaffolds by a three-component reaction with 1,3-dicarbonyls. Synlett 2007, 1323-1325.

8. (a) Multicomponent reactions: concepts and applications for design and synthesis, Herrera, R. P.; Marqués-López, E., Eds.; Wiley: Hoboken, 2015. (b) Multicomponent reactions in organic synthesis, Zhu, J.; Wang, Q.; Wang, M.-X., Eds.; Wiley-VCH: Weinheim, 2014.
9. (a) Ramazani, A.; Rezaei, A. Novel one-pot, four-component condensation reaction: an efficient approach for the synthesis of 2,5-disubstituted 1,3,4-oxadiazole derivatives by a Ugi4CR/aza-Wittig sequence, Org. Lett. 2010, 12, 2852-2855. (b) Dömling, A. Recent developments in isocyanide based multicomponent reactions in applied chemistry, Chem. Rev. 2006, 106, 17-89. (c) Dömling, A.; Ugi, I. Multicomponent reactions with isocyanides, Angew. Chem. Int. Ed. 2000, 39, 3168-3210.

10. (a) De Moliner, F.; Hulme, C. Straightforward assembly of phenylimidazoquinoxalines via a one-pot two-step MCR process. Org. Lett. 2012, 14, 1354-1357. (b) Dömling, A.; Huang, Y. Piperazine scaffolds via isocyanide-based Multicomponent Reactions. Synthesis 2010, 2859-2883.

11. (a) Stiernet, P.; Lecomte, P.; De Winter, J.; Debuigne, A. Ugi three-component polymerization toward poly $(\alpha$-amino amide)s. ACS Macro Lett. 2019, 8, 427-434. (b) Llevot, A.; Boukis, A.C.; Oelmann, S.; Wetzel, K.; Meier, M. A. R. An update on isocyanide-based Multicomponent Reactions in polymer science. Top. Curr. Chem. 2017, 375, 66. (c) Hartweg, M.; Becer, C. R. Direct polymerization of levulinic acid via Ugi multicomponent reaction. Green Chem. 2016, 18, 3272-3277. (d) Sehlinger, A.; Dannecker, P.; Kreye, O.; Meier, M. A. R. Diversely substituted polyamides: Macromolecular design using the Ugi four-component reaction. Macromolecules 2014, 47, 2774-2783. (e) Sehlinger, A.; Schneider, R.; Meier, M. A. $\mathrm{R}$. Ugi reactions with $\mathrm{CO} 2$ : Access to functionalized polyurethanes, polycarbonates, polyamides, and Polyhydantoins. Macromol. Rapid Commun. 2014, 35, 18661871

12. (a) Dannecker, P.-K.; Sehlinger, A.; Meier, M. A. R. Polymacrocycles derived via Ugi multi-component reactions. Macromol. Rapid Commun. 2019, 40, 1800748. (b) Wessjohann, A.; Voigt, B.; Rivera, D. G. Diversity oriented one-pot synthesis of complex macrocycles: Very large steroidpeptoid hybrids from multiple multicomponent reactions including bifunctional building blocks. Angew. Chem. Int. Ed. $\mathbf{2 0 0 5}, 44,4785-4790$

13. (a) Azuaje, J.; El Maatougui, A.; García-Mera, X.; Sotelo, E. Ugi-based approaches to quinoxaline libraries. ACS Comb. Sci. 2014, 16, 403-411. (b) Hulme, C.; Peng, J.; Louridas, B.; Menard, P.; Krolikowski, P.; Kumar, N. V. Applications of NBOC-diamines for the solution phase synthesis of ketopiperazine libraries utilizing a Ugi/De-BOC/cyclization (UDC) strategy. Tetrahedron Lett. 1998, 39, 8047-8050.

14. (a) Kysil, V.; Khvat, A.; Tsirulnikov, S.; Tkachenko, S.; Willians, C.; Churakova, M.; Ivachtchenko, A. General multicomponent strategy for the synthesis of 2-amino-1,4diazaheterocycles: Scope, limitations, and utility. Eur. J. Org Chem. 2010, 1525-1543. (b) Shaabani, A.; Maleki, A.; Moghimi-Rad, J. A novel isocyanide-based three-component reaction: Synthesis of highly substituted 1,6-dihydropyrazine2,3-dicarbonitrile derivatives. J. Org. Chem. 2007, 72, 63096311.

15. Giovenzana, G. B.; Tron, G. C.; Di Paola, S.; Menegotto, I. G.; Pirali, T. A mimicry of primary amines by bis-secondary diamines as components in the Ugi four-component reaction. Angew. Chem. Int. Ed. 2006, 45, 1099-1102.

16. (a) Shaabani, A.; Hajishaabanha, F.; Mahyari, M.; Mofakham, $\mathrm{H}$.; $\mathrm{Ng}$, S. W. A novel one-pot pseudo-four-component isocyanide-based reaction: an unexpected approach for the synthesis of tetrahydrodiisoindoloquinoxalines and tetrahydrobenzodiisoindoloquinoxalines. Tetrahedron, 2011, 67, 8360-8366. (b) Hulme, C.; Cherrier, M. -P. Novel applications of ethyl glyoxalate with the Ugi MCR. Tetrahedron Lett. 1999, 40, 5295-5299. (c) Zychlinski, A. V.; Ugi, I. MCR IX: A new and easy way for the preparation of piperazine-2-keto-3-carboxamides. Heterocycles 1998, 49, 2932. (d) Rossen, K.; Sager, J.; DiMichele, L. M. An efficient and versatile synthesis of piperazine-2-carboxamides. Tetrahedron Lett. 1997, 38, 3183-3186. 
17. Marcaccini, S.; Torroba, T. The use of the Ugi four-component condensation. Nat. Protoc. 2007, 2, 632-639.

18. Li, Y.; He, Y.; Chen, F.; Fan, Q. Asymmetric hydrogenation of 2-aryl-5,6-dihydropyrazine derivatives with chiral cationic ruthenium diamine catalysts. Chin. J. Chem. 2014, 32, 991994.

19. Stork, G.; Terrell, R.; Szmuszkovicz, J. A new synthesis of 2alkyl and acyl ketones. J. Am. Chem. Soc. 1954, 76, 2029-2030.

20. Pertejo, P.; Peña-Calleja, P.; Carreira-Barral, I.; Quesada, R.; Cordero, N. A.; Rodríguez, F. J.; García-Valverde, M. Novel pyrrolobenzodiazepine and pyrroloquinazoline scaffolds synthesized by a simple and highly selective Ugi/cyclization sequence. Org. Biomol. Chem., 2017, 15, 7549-7557.
21. Pertejo, P.; Corres, N.; Torroba, T.; García-Valverde, M. Reversal of diastereoselectivity in the synthesis of peptidomimetic 3 - carboxamide-1,4-benzodiazepin-5-ones. Org. Lett. 2015, 17, 612-615.

22. Banfi, L.; Basso, A.; Guanti, R.; Riva, R. Asymmetric Isocyanide-Based MCRs. In Multicomponent Reactions; Zhu, J. P.; Bienaymé, H., Eds.; Wiley: Weinheim, 2005; pp 1-32.

23. X-Ray analysis for $7 \mathbf{a}$ was carried out on the racemic form of 7a synthesized from ( \pm -1,2-cyclohexyldiamine.

24. Frisch, M. J.; Trucks, G. W. et al; GAUSSIAN 09 program package, Gaussian Inc., Wallingford CT, 2009. 